\title{
Imaging Features of Nonenhancing Diffuse Midline Gliomas (DMGs) with Histone H3 K27M Mutation: Spectroscopy and Perfusion Imaging Findings
}

\author{
Ozum Tuncyurek' (D), Mehmet Alp Dirik² (D), Nail Bulakbaşı ${ }^{2}$ (D) \\ 'Department of Radiology, Near East University Faculty of Medicine, Northern Cyprus, Turkey \\ ${ }^{2}$ Department of Radiology, Dr. Suat Günsel University of Kyrenia Hospital, Northern Cyprus, Turkey \\ ORCID iDs of the authors: O.T. 0000-0003-I669-082X; M.A.D. 0000-000I-9427-282I; N.B. 0000-000I-7528-8107.
}

Cite this article as: Tuncyurek O, Dirik MA, Bulakbaşı N. Imaging Features of Nonenhancing Diffuse Midline Gliomas (DMG) with Histone H3 K27M Mutation: Spectroscopy and Perfusion Imaging Findings. Cyprus J Med Sci. 2021; 6(3): 265-267.

\begin{abstract}
Diffuse midline gliomas (DMGs) with the histone H3 K27 mutation is a brand-new tumor according to the 2016 World Health Organization Classification of Tumors of the Central Nervous System. In this case report, we characterize the magnetic resonance imaging (MRI) aspects with the literature review. Instead of biopsy, MRI techniques are used to diagnose the tumor and determine the treatment choice despite the correlation with the prognosis. We present the case of high-grade astrocytic tumors with $\mathrm{H} 3 \mathrm{~K} 27$ mutation in a 19 year-old woman. MRI revealed a DMG. There was no paralysis of extremities or cerebellar deficit detected in physical examination. MRI showed a tumor in the pons, which is a hypo intense on Tl-weighted imaging and a hyper intense on T2-weighted imaging. A nonenhancing lesion was discovered during a Gd-MRI examination. In perfusion imaging, there was no distinct perfusion rising in the tumor. $\mathrm{N}$-Acetyl-aspartate (NAA):Cr ratio was 0.57 , and choline (Cho):Cr ratio was 0.78 . In conclusion, perfusion and spectroscopy findings are helpful for nonenhancing DMG diagnosis.
\end{abstract}

Keywords: Diffuse midline glioma, perfusion MRI, spectroscopy

\section{INTRODUCTION}

Being classified as a new type of tumor in the World Health Organization 2016 Edition of Tumors of the Central Nervous System, the diffuse midline glioma (DMG), H3 K27M mutant, manifests in the thalamus, brain stem, and spinal cord of children and adolescent.' Castel et al. ${ }^{2}$ note that the occurrence of each mutation varies based on the primary location. They demonstrate how the frequency of H3.3 K27M mutation in all thalamic gliomas cases tripled the rate of the H3.I K27M mutation. Proving the point, Nakata et al. ${ }^{3}$ detected $20 \%$ H3F3A K27M mutant gliomas among 10 adults with cerebellar high-grade gliomas.

In this paper, we introduce an uncommon case of young person pontine high-grade DMG with H3 K27M mutation.

\section{CASE PRESENTATION}

A 19-year-old woman was examined in an ear-nose-throat outpatient clinic with a complaint of hearing loss in the last 2 months. After obtaining an informed consent, a DMG was found through magnetic resonance imaging (MRI) (Figure I). There was no paralysis of extremities or no cerebellar deficit in the physical examination. MRI showed a tumor involving in the right mesencephalon, pons, and superior bulbous. It was enlarging the brainstem and compressing the fourth ventricle. The tumor was also noted to have a hypointensity on TI- and hyperintensity on T2-weighted images with T2/FLAIR mismatch. Following the gadolinium-based contrast material injection, the tumor did not enhance (Figure l a-d), and no cystic lesions were present. The tumor had a minimum Apparent diffusion coefficient (ADC) value of $1.41 \times 10^{-3} \mathrm{~mm}^{2} \mathrm{~s}^{-1}$, kurtosis value of 532, choline (Cho) /Cr ratio of $0.98, \mathrm{~N}$-acetyl-aspartate (NAA)/Cr ratio of 0.57 , and relative cerebral blood volume (rCBV) value of 0.78 .

\section{DISCUSSION}

DMG is a rare infiltrative astrocytoma centered in the pons and is a highly malignant tumor that affects children and adolescents. They have a bad prognosis with most of the patients deceasing within a year from diagnosis. ${ }^{4}$ In the 


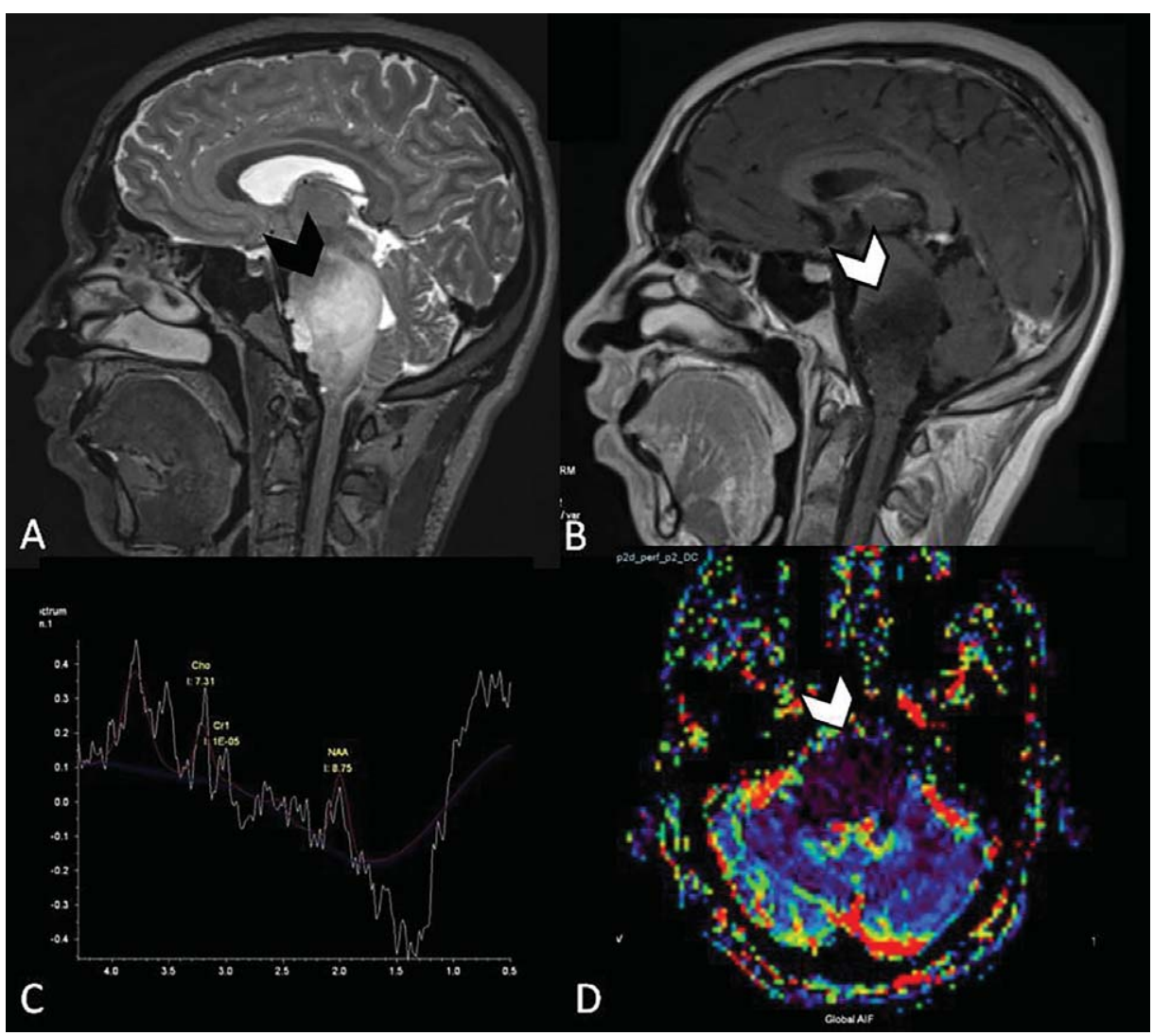

Figure I. a-d. (a) Sagittal T2-weighted magnetic resonance imaging (MRI-MAGNETOM Aera I.5T, Siemens, Erlangen, Germany) shows the mass in pons (black arrowhead). (b) Contrast-enhanced sagittal MRI shows a nonenhancement mass (white arrowhead). (c) Axial multivoxel MR spectroscopy shows the low NAA. (d) Perfusion-CBV map image shows low perfusion values (white arrowhead).

United States, approximately 100-150 children are diagnosed annually with DMG. ${ }^{5}$

Surgery and radiotherapy are limited solutions for the treatment. DMG diagnosis has traditionally been made on clinical grounds based on characteristic symptoms and MRI findings.

Imaging features of H3 K27M mutant gliomas were the absence of heterogeneous contrast enhancement and necrosis in half of cases. ${ }^{6}$ Pons gliomas are a variable degree of contrast enhancement and uniformly enhancement in the spine. Cervical spine gliomas show cerebrospinal fluid-based spread. The thalamic and pontine gliomas demonstrated local recurrence.

Although DMG diagnosis is mostly established by examining its radiographic and clinical features, stereotactic biopsy is a minimal morbid procedure and allows for a definitive histopathological classification. ${ }^{7,8}$ The standard radiotherapy is available for treatment in children. 90

Two major conclusions have been drawn out from numerous studies on the topic: DMGs molecular features differ from those of adult and pediatric supratentorial hemispheric gliomas, and distinct subcategories with differing genetic, epigenetic, and proteomic makeup attributes exist within DMG classification."

The variations among the location of gliomas in pediatric and adult patients may be a substantial determiner of tumor act and morphology. Gliomas are most typically encountered within the hemispheres or midline structures of supratentorial region in adult patients, whereas in children, infratentorial lesions are more commonly confined to the brainstem. Generally, brainstem gliomas produce only $1 \%$ of adult high-grade gliomas compared with $10 \%$ of pediatric gliomas. ${ }^{12}$

In pediatric gliomas, the various mutations in histone 3 genes are based on their varying locations. While H3.I K27M and H3.3 K27M mutations are frequently encountered in patients with DMG and midline nonbrainstem high-grade gliomas, the $\mathrm{H} 3.3$ G34R/ $\vee$ mutation is solely observed in hemispheric high-grade gliomas. $^{3}$

Due to the scarcity of standardization, the impact spectroscopy on patient control has been ambiguous. In our case, Cho:NAA ratio was 0.8 in the center, and variation was high. Increased Cho:NAA ratio variation in mass voxels determined by multivoxel spectroscopy can give information about poor clinical prognosis. ${ }^{14}$ Cho is an integral member of cell membranes, while NAA is mainly in normal neurons. Elevated Cho:NAA can be associated with an increased number of cells, cell turnover, and injured cells. Steffen-Smith and coworkers's $s^{15}$ published study identified a threshold value; Cho:NAA was 2.I that allows classification of patients by risk. Cho:NAA was 0.8 in our patient. Patients with a lesser value have a significantly higher survival chance compared with patients who had at least one examination with recorded higher values. 
Increased rCBV values in DMG were found to be associated with mortality rate in one study. ${ }^{15}$ Although quite a few rCBV cutoff values to predict poor or more favorable outcome without histopathological grading have been published, the most widely accepted is that of 1.75 , projected by Law et al. ${ }^{16}$ in adult gliomas. In our patient, rCBV value was 0.78 .

In conclusion, imaging features of $\mathrm{H} 3 \mathrm{~K} 27 \mathrm{M}$ mutant gliomas were the absence or heterogeneous contrast enhancement and necrosis in half of cases. Perfusion and spectroscopy findings of DMG are helpful for diagnosis.

Informed Consent: Written informed consent was obtained from all participants who participated in this study.

Peer-review: Externally peer-reviewed.

Author Contributions: Conception - O.T.; Supervision - N.B.; Materials O.T.; Data Collection and/or Processing - O.T.; Analysis and/or Interpretation - N.B.; Literature Review - M.D.; Writing - O.T.; Critical Reviews - O.T., M.D., N.B.

Conflict of Interest: The authors have no conflicts of interest to declare.

Financial Disclosure: The authors declared that this study has received no financial support.

\section{REFERENCES}

I. Johnson DR, Guerin JB, Giannini C, Morris JM, Eckel LJ, Kaufmann TJ. Updates to the WHO brain tumor classification system: What the radiologist needs to know. Radiographics. 2017;37(7):2164-2180. [CrossRef]

2. Castel D, Philippe C, Kergrohen T, et al. Transcriptomic and epigenetic profiling of 'diffuse midline gliomas, H3 K27M-mutant' discriminate two subgroups based on the type of histone $\mathrm{H} 3$ mutated and not supratentorial or infratentorial location. Acta Neuropathol Commun. 2018;6(I):117. [CrossRef]

3. Nakata S, Nobusawa S, Yamazaki T, et al. Histone H3 K27M mutations in adult cerebellar high-grade gliomas. Brain Tumor Pathol. 2017;34(3):I13-119. [CrossRef]

4. Buczkowicz P, Hawkins C. Pathology, molecular genetics, and epigenetics of diffuse intrinsic pontine glioma. Front Oncol. 2015;5:I-9. [CrossRef]
5. Smith M, Freidlin B, Ries LA, Simon R. Trends in reported incidence of primary malignant brain tumors in children in the United States. J Natl Cancer Inst. 1998;90:1269-1277. [CrossRef]

6. Hankinson TC, Campagna EJ, Forman NK, Handler MH. Interpretation of magnetic resonance images in diffuse intrinsic pontine glioma: A survey of pediatric neurosurgeons. J Neurosurg Pediatr. 2011;8:97-102. [CrossRef]

7. Roujeau T, Machado G, Garnett MR, et al. Stereotactic biopsy of diffuse pontine lesions in children. J Neurosurg. 2007;107(Suppl. I):I4. [CrossRef]

8. Cage TA, Samagh SP, Mueller S, et al. Feasibility, safety, and indications for surgical biopsy of intrinsic brainstem tumors in children. Childs Nerv Syst. 2013;29:1313-1319. [CrossRef]

9. Kebudi R, Cakir FB. Management of diffuse pontine gliomas in children: Recent developments. Pediatr Drugs. 2013;15:351-362. [CrossRef]

10. Zaghloul MS, Eldebawy E, Ahmed S, et al. Hypofractionated conformal radiotherapy for pediatric diffuse intrinsic pontine glioma (DIPG): A randomized controlled trial. Radiother Oncol. 2014;III:3540. [CrossRef]

II. Saratsis A, Kambhampati M, Snyder K, et al. Comparative multidimensional molecular analyses of pediatric diffuse intrinsic pontine glioma reveals distinct molecular subtypes. Acta Neuropathol. 2014;127:881-895. [CrossRef]

12. Rineer J, Schreiber D, Choi K, Rotman M. Characterization and outcomes of infratentorial malignant glioma: A population-based study using the surveillance epidemiology and end-results database. Radiother Oncol. 2010;95:321-326. [CrossRef]

13. Wu G, Diaz AK, Paugh BS, et al. The genomic landscape of diffuse intrinsic pontine glioma and pediatric non-brainstem high-grade glioma. Nat Genet. 2014;46:444-450.

14. Steffen-Smith EA, Venzon DJ, Bent RS, Hipp SJ, Warren KE. Singleand multivoxel proton spectroscopy in pediatric patients with diffuse intrinsic pontine glioma. Int J Radiat Oncol Biol Phys. 2012;84(3):774-779. [CrossRef]

15. Hipp SJ, Steffen-Smith E, Hammoud D, Shih JH, Bent R, Warren KE. Predicting outcome of children with diffuse intrinsic pontine gliomas using multiparametric imaging. Neuro Oncol. 2011;13(8):904909. [CrossRef]

16. Law M, Yang S, Wang $H$, et al. Glioma grading: Sensitivity, specificity, and predictive values of perfusion MR imaging and proton MR spectroscopic imaging compared with conventional MR imaging. AJNR Am J Neuroradiol. 2003;24(10):1989-1998. 